\title{
Layered composite and contact layer. Normal separation and transversal strength
}

\author{
Vladimir Andreev*, Robert Turusov and Nikita Tsybin \\ Moscow State University of Civil Engineering, Yaroslavskoe shosse, 26, Moscow, 129337, Russia
}

\begin{abstract}
As well as testing adhesion joints, in which the average adhesion shear strength is measured, there are widespread methods of testing for normal separation, in which the value called transversal strength is measured. This value is calculated as the ratio of the breaking load applied perpendicular to the gluing plane, to the gluing area. At the same time, two types of tests for normal separation - for cylindrical and prismatic joints - are now standardized. The article deals with the solution of this problem for prismatic compound obtained on the basis of the contact layer model.
\end{abstract}

\section{Statement of the problem}

Adhesion is the phenomenon of the emergence and establishment of bonds between the adhesive and the substrate. The intensity of the adhesive interaction is determined by the energy of the bonds established and their density. The density of atoms on the surface of the crystal is known to be approximately $10^{14} / \mathrm{cm}^{2}$. In this case, the density of the bonds established $10^{13} / \mathrm{cm}^{2}$ will mean that approximately every tenth atom from the surface area of the substrate with the opposing atom of the adhesive is involved in the adhesive interaction. With a bond density of $10^{12} / \mathrm{cm}^{2}$ every hundredth atom is involved. Since the energy of one bond in the cases considered is the same, it follows that the intensity of the adhesive interaction can vary in the range of several orders of magnitude.

The contact layer model proposes to describe the medium in which interatomic bonds between the adhesive and substrate layers arise from the point of view of solid mechanics. It is assumed that the surface density of the bonds does not exceed $10^{-1}$ of the density of atoms on the contact surface, and the length of these bonds is $h^{*}$. This assumption allows us to consider the contact layer as a transversally anisotropic elastic medium [1]. The parameters of this medium are such that it can be represented as a thick brush of elastic rods, not connected to each other and oriented normally to the contact surface. In such a medium, there are no normal stresses in the contact plane (perpendicular to the "lateral" surface of the rods), and the Poisson's ratio (since the rods are not connected) is zero. As a result, the main characteristics of the contact layer to be determined from the macro experiment are its thickness $h^{*}$ and shear stiffness $G^{*}$, and since the Poisson's ratio is assumed to be zero, the shear modulus is associated with the Young's modulus by a simple

Corresponding author: asv@mgsu.ru 
equation $G^{*}=E^{*} / 2$. The basic relations of the theory of elasticity of anisotropic bodies (equilibrium equations, Hooke's law and Cauchy relations) remain unchanged.

A general view of a prismatic layered composite with contact layers under conditions of normal separation is shown in Figure 1.

We denote the hypotheses that will be used in the derivation of resolving equations for the problem:

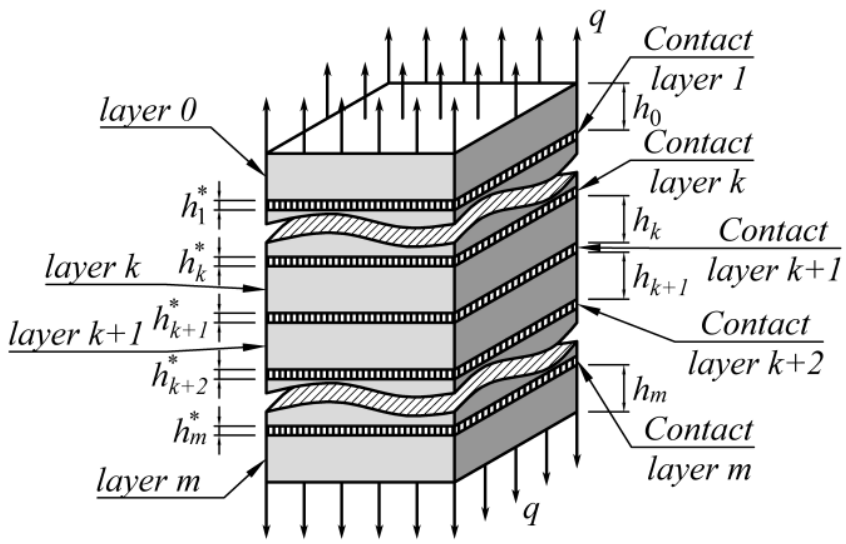

1) The adhesive and substrate layers are isotropic and obey Hooke's law.

2) The interaction of the adhesive and substrate layers is implemented through a contact layer.

3) The loading scheme of the model assumes that the lateral faces are free from loads. A tensile stress $q$ is applied to the top and bottom faces.

Fig 1. Model of layered composite

4) It is assumed that the stiffness of the bonded substrate layers is such that the bending can be neglected. As a result, the stresses $\sigma_{z}$ are constant throughout the model and equal to the value of the applied tensile load, i.e. $\sigma_{z}=q$.

5) Since there is no bending, deformations $\varepsilon_{x}$ and $\varepsilon_{y}$ of layers of adhesive and substrate, displacements $u$ and $v$, as well as stresses $\sigma_{x}$ and $\sigma_{y}$ are functions of two variables $x$ and $y$.

6) It is assumed that there are only elastic deformations in each layer $k$ of the composite.

\section{System of resolving equations}

The physical side of the problem follows from Hooke's law for the layer $k$ of adhesive or substrate (hereinafter simply layer $k$ ). The equations that determine the relationship between stresses and strains are written below.

$$
\left.\begin{array}{l}
\varepsilon_{x, k}=\frac{1}{E_{k}}\left[\sigma_{x, k}-v_{k}\left(q+\sigma_{y, k}\right)\right] ; \\
\varepsilon_{y, k}=\frac{1}{E_{k}}\left[\sigma_{y, k}-v_{k}\left(q+\sigma_{x, k}\right)\right] ; \\
\varepsilon_{z, k}=\frac{1}{E_{k}}\left[q-v_{k}\left(\sigma_{x, k}+\sigma_{y, k}\right)\right] .
\end{array}\right\}
$$

The static side of the problem follows from the equilibrium of the layer $k$. In Figure 2, the contact layers adjacent to the layer $k$ are replaced by the shear stresses acting in them. 


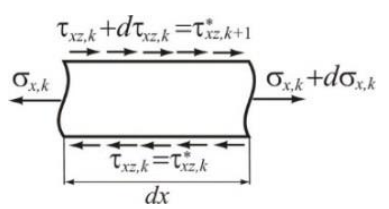

Fig. 2. Layer $k$ equilibrium

Analyzing Figure 2 and taking into account that the stresses due to the absence of bending do not vary in thickness; we write the equilibrium conditions of any layer $k$ of the composite:

$$
\tau_{x z, k}^{*}-\tau_{x z, k+1}^{*}=h_{k} \frac{\partial \sigma_{x, k}}{\partial x} ; \quad \tau_{y z, k}^{*}-\tau_{y z, k+1}^{*}=h_{k} \frac{\partial \sigma_{y, k}}{\partial y} .
$$

Let us consider the contact layer. In [2] the resolving equations for the contact layer were obtained. Since there is no bending in the adhesive and substrate layers for the contact layer model of the general form, these equations are written in the form:

$$
\left.\begin{array}{c}
\tau_{x z, k}^{*}=\frac{\left(h_{k}^{*}\right)^{2}}{24} \frac{\partial}{\partial x}\left(\frac{\partial \tau_{x z, k}^{*}}{\partial x}+\frac{\partial \tau_{y z, k}^{*}}{\partial y}\right)-\frac{G_{k}^{*}}{h_{k}^{*}}\left(u_{k-1}-u_{k}\right) ; \\
\tau_{y z, k}^{*}=\frac{\left(h_{k}^{*}\right)^{2}}{24} \frac{\partial}{\partial y}\left(\frac{\partial \tau_{y z, k}^{*}}{\partial y}+\frac{\partial \tau_{x z, k}^{*}}{\partial x}\right)-\frac{G_{k}^{*}}{h_{k}^{*}}\left(v_{k-1}-v_{k}\right) .
\end{array}\right\}
$$

These equations are obtained by directly integrating of the equations of the theory of elasticity of anisotropic bodies, taking into account the hypotheses used for the contact layer. The boundary conditions are the continuity of the displacement vector at the interface with the adjacent layers.

Equations (3) assume a nonlinear distribution of displacements over the thickness of the contact layer. Considering the simplified model of the contact layer (the linear law of the distribution of displacements over the thickness of the contact layer), we turn to the linearized equations, in which the bending can also be neglected. As a result, we obtain:

$$
\left.\begin{array}{c}
\frac{\tau_{x z, k}^{*}}{G_{k}^{*}}=-\frac{1}{h_{k}^{*}}\left(u_{k-1}-u_{k}\right) \\
\frac{\tau_{y z, k}^{*}}{G_{k}^{*}}=-\frac{1}{h_{k}^{*}}\left(v_{k-1}-v_{k}\right) .
\end{array}\right\}
$$

These equations are also obtained in [3] and [4].

For the model of the contact layer of the general form, equations (1), (2) and (3) can be reduced to a system of two equations with respect to stresses $\sigma_{x, k}^{f}$ and $\sigma_{y, k}^{f}$

$$
\left.\begin{array}{rl}
h_{k} \frac{\partial^{2} \sigma_{x, k}^{f}}{\partial x^{2}} & =h_{k} \frac{\left(h^{*}\right)^{2}}{24} \frac{\partial^{2}}{\partial x^{2}}\left(\frac{\partial^{2} \sigma_{x, k}^{f}}{\partial x^{2}}+\frac{\partial^{2} \sigma_{y, k}^{f}}{\partial y^{2}}\right)- \\
- & \frac{G^{*}}{h^{*}}\left(\varepsilon_{x, k-1}^{f}-2 \varepsilon_{x, k}^{f}+\varepsilon_{x, k+1}^{f}\right) ; \\
h_{k} \frac{\partial^{2} \sigma_{y, k}^{f}}{\partial y^{2}} & =h_{k} \frac{\left(h^{*}\right)^{2}}{24} \frac{\partial^{2}}{\partial y^{2}}\left(\frac{\partial^{2} \sigma_{y, k}^{f}}{\partial y^{2}}+\frac{\partial^{2} \sigma_{x, k}^{f}}{\partial x^{2}}\right)- \\
- & \frac{G^{*}}{h^{*}}\left(\varepsilon_{y, k-1}^{f}-2 \varepsilon_{y, k}^{f}+\varepsilon_{y, k+1}^{f}\right) .
\end{array}\right\}
$$

The index $f$ here means that the model of the contact layer of the general form is considered. Components of the deformations $\varepsilon_{x, i}^{f}$ and $\varepsilon_{y, i}^{f}$ were recorded earlier (1).

A similar (5) system of equations can be written for a simplified model of the contact layer 


$$
\left.\begin{array}{l}
h_{k} \frac{\partial^{2} \sigma_{x, k}^{s}}{\partial x^{2}}=-\frac{G^{*}}{h^{*}}\left(\varepsilon_{x, k-1}^{s}-2 \varepsilon_{x, k}^{s}+\varepsilon_{x, k+1}^{s}\right) \\
h_{k} \frac{\partial^{2} \sigma_{x, k}^{s}}{\partial y^{2}}=-\frac{G^{*}}{h^{*}}\left(\varepsilon_{y, k-1}^{s}-2 \varepsilon_{y, k}^{s}+\varepsilon_{y, k+1}^{s}\right) .
\end{array}\right\}
$$

The index $s$ means that a simplified model of the contact layer is considered.

\section{Three-layer model}

Let us consider a three-layer composite consisting of two identical layers of substrate,

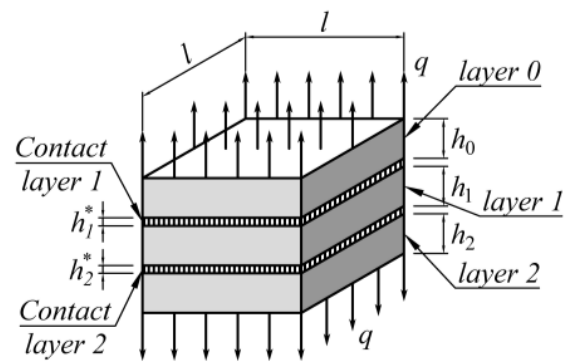

Fig. 3. Model with three layers. bonded with adhesive (Figure 3). For simplicity, the section will be considered square with dimensions $l \times l$.

Since the cross section is square, in equations (1), (5) and (6) it is necessary to take into account the fact that at points with the same values $x, y$ the stresses $\sigma_{x, k}$ and $\sigma_{y, k}$ are equal.

Due to the fact that the layers of the substrate are the same, we assume:

$$
E_{0}=E_{2}=E_{s} ; v_{0}=v_{2}=v_{s} ; h_{0}=h_{2}=h_{s} ; h_{1}=h_{a} ; E_{1}=E_{a} ; v_{1}=v_{a} \text {. }
$$

From the equilibrium condition of the composite as a whole, we obtain the following relations:

$$
\sigma_{x, 0} h_{0}=\sigma_{x, 2} h_{2}=-\frac{1}{2} \sigma_{x, 1} h_{1} \rightarrow \sigma_{x, 0}=\sigma_{x, 2}=-\frac{\sigma_{x, 1} h_{a}}{2 h_{s}} ; \tau_{x z, 1}^{*}=-\tau_{x z, 2}^{*}=\tau^{*} .
$$

From equality (2), taking into account the last expression (8), we find the shear stresses in the contact layer.

$$
\tau_{x z, 1}^{*}-\tau_{x z, 2}^{*}=2 \tau^{*}=h_{1} \frac{d \sigma_{x, 1}}{d x} \rightarrow \tau^{*}=\frac{h_{a}}{2} \frac{d \sigma_{x, 1}}{d x} .
$$

As a result, the systems of resolving equations can be reduced to the equations for the stresses $\sigma_{x, 1}$ in the adhesive layer:

in which

$$
\left.\begin{array}{c}
\frac{d^{2} \sigma_{x, 1}^{s}}{d x^{2}}-\sigma_{x, 1}^{s} \omega^{2}+q \lambda_{q}^{2}=0 \\
-2 \eta \frac{d^{2} \sigma_{x, 1}^{f}}{d x^{2}}+2 \sigma_{x, 1}^{f} \eta \omega^{2}-2 q \eta \lambda_{q}^{2}=0
\end{array}\right\}
$$

$$
\omega^{2}=\frac{G^{*}}{h_{a} h^{*}}\left[\frac{2\left(1-v_{a}\right)}{E_{a}}+\frac{h_{a}}{h_{s}} \frac{\left(1-v_{s}\right)}{E_{s}}\right] ; 2 \eta=\frac{12}{\left(h^{*}\right)^{2}} ; \lambda_{q}^{2}=\frac{2 G^{*}}{h_{a} h^{*}}\left(\frac{v_{a}}{E_{a}}-\frac{v_{s}}{E_{s}}\right) .
$$

The general solutions of equations (10) are written below.

$$
\left.\begin{array}{c}
\sigma_{x, 1}^{s}=\frac{q \lambda_{q}^{2}}{\omega^{2}}+C_{1}^{s} \exp (\omega x)+C_{2}^{s} \exp (-\omega x) ; \\
\sigma_{x, 1}^{f}=\frac{q \lambda_{q}^{2}}{\omega^{2}}+C_{1}^{f} \exp \left(\psi_{1}^{f} x\right)+C_{2}^{f} \exp \left(-\psi_{1}^{f} x\right)+C_{3}^{f} \exp \left(\psi_{2}^{f} x\right)+C_{4}^{f} \exp \left(-\psi_{2}^{f} x\right),
\end{array}\right\}
$$


in which $\psi_{1}^{f}=\sqrt{\eta-\sqrt{\eta^{2}-2 \omega^{2} \eta}} ; \psi_{2}^{f}=\sqrt{\eta+\sqrt{\eta^{2}-2 \omega^{2} \eta}}$. The indices $s$ and $f$ mean that the solution is written for a simplified and general model of the contact layer respectively.

The unknown integration constants included in equations (12), are determined from the boundary conditions written below

$$
\sigma_{x, 1}^{s, f}\left( \pm \frac{l}{2}\right)=0 ; \tau^{* f}\left( \pm \frac{l}{2}\right)=\frac{h_{a}}{2} \frac{d \sigma_{x, 1}^{f}}{d x}\left( \pm \frac{l}{2}\right)=0 .
$$

From the second expression (13) it follows that the shear stresses at the lateral end of the model are zero. Using the boundary conditions (13), we find

$$
\begin{aligned}
& \sigma_{x, 1}^{s}=\frac{q \lambda_{q}^{2}}{\omega^{2}}\left[1-\frac{\cosh (\omega x)}{\cosh (\omega l / 2)}\right] ; \\
& \sigma_{x, 1}^{f}=\frac{q \lambda_{q}^{2}}{\omega^{2}}\left[1-\frac{\psi_{1}^{f} \sinh \left(\frac{\psi_{1}^{f} l}{2}\right) \cosh \left(\psi_{2}^{f} x\right)-\psi_{2}^{f} \sinh \left(\frac{\psi_{2}^{f} l}{2}\right) \cosh \left(\psi_{1}^{f} x\right)}{\psi_{1}^{f} \sinh \left(\frac{\psi_{1}^{f} l}{2}\right) \cosh \left(\frac{\psi_{2}^{f} l}{2}\right)-\psi_{2}^{f} \sinh \left(\frac{\psi_{2}^{f} l}{2}\right) \cosh \left(\frac{\psi_{1}^{f} l}{2}\right)}\right] .
\end{aligned}
$$
layer:

From equality (9) using the expressions (14) we find the shear stresses in the contact

$$
\begin{gathered}
\tau^{* s}=-\frac{h_{a}}{2} \frac{q \lambda_{q}^{2}}{\omega} \frac{\sinh (\omega x)}{\cosh (\omega l / 2)} \\
\tau^{* f}=-\frac{h_{a}}{2} \frac{q \lambda_{q}^{2}}{\omega^{2}} \frac{\psi_{1}^{f} \psi_{2}^{f} \sinh \left(\frac{\psi_{1}^{f} l}{2}\right) \sinh \left(\psi_{2}^{f} x\right)-\psi_{1}^{f} \psi_{2}^{f} \sinh \left(\frac{\psi_{2}^{f} l}{2}\right) \sinh \left(\psi_{1}^{f} x\right)}{\psi_{1}^{f} \sinh \left(\frac{\psi_{1}^{f} l}{2}\right) \cosh \left(\frac{\psi_{2}^{f} l}{2}\right)-\psi_{2}^{f} \sinh \left(\frac{\psi_{2}^{f} l}{2}\right) \cosh \left(\frac{\psi_{1}^{f} l}{2}\right)} .
\end{gathered}
$$

\section{Results of calculation}

Let us compare the normal and shear stresses acting in the adhesive layer calculated by formulae (14) and (15).
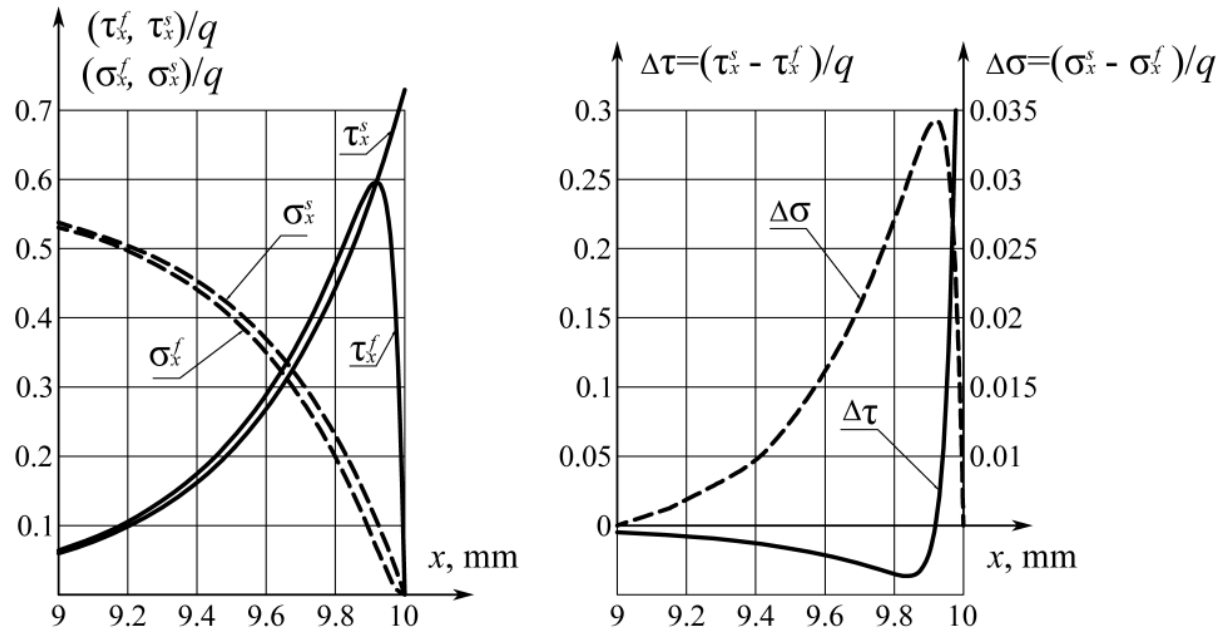

Fig. 4. Results of stress calculation in the adhesive layer (left). The error of calculation of stresses (right). 
The following parameters are used in the calculation:

$$
\begin{gathered}
h_{s}=10 \mathrm{~mm} ; h_{a}=1 \mathrm{~mm} ; l=20 \mathrm{~mm} ; E_{s}=2 \cdot 10^{5} \mathrm{MPa} ; \\
E_{a}=2 \cdot 10^{3} \mathrm{MPa} ; v_{s}=0.3 ; v_{a}=0.37 ; \frac{G^{*}}{h^{*}}=10^{4} \mathrm{MPa} / \mathrm{mm} .
\end{gathered}
$$

From Figure 4 it follows that the simplified model of the contact layer does not allow to completely satisfy all boundary conditions (equality to zero of the shear stresses at the free

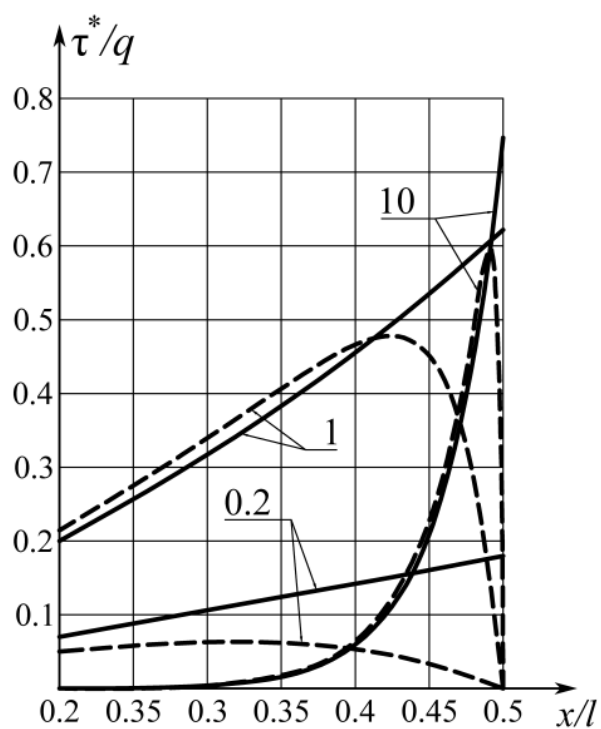

Fig. 5. Distribution of shear stresses along the bonding length for different length values. Solid lines - simplified contact layer model. Dashed lines - general form contact layer model. The captions of the diagrams correspond to values of length $(l, \mathrm{~mm})$ used in the calculation. edge) in contrast to the model of the contact layer in a general form. The width of the edge effect zone in the two models is almost equal. This fact should be taken into account by processing experimental data. The maximum shear stress value for the selected parameters (using the simplified model) exceeds the same value for the contact layer model of the general form. The normal stresses for the two models under consideration are almost equal.

One of the basic parameters in the tests of adhesive compounds at normal separation is the bounding area. A series of graphs in Figure 5 allows estimating the effect of length $l$ on shear stresses in the contact layer for two different contact layer models.

Considering Figure 5, we conclude that decreasing of the length of the gluing leads to shear stresses decreasing. In the limit $l \rightarrow 0$, the shear stresses in the contact layer tend to zero for both models.

We will consider a simplified model of the contact layer for further analysis. This choice is due to the fact that it is not possible to determine analytically the point with maximum shear stresses for the general form model. For simplified model shear stresses reach their maximum value at point $x= \pm l / 2$ and are equal

$$
\tau_{\max }^{*}=-\frac{h_{a}}{2} \frac{q \lambda_{q}^{2}}{\omega} \tanh \left(\frac{\omega l}{2}\right) .
$$

If we denote a certain value $\left|\tau_{\max }^{*}\right|=\tau_{\text {adh }}$ at which the shear fracture occurs, from formula (16) we can find an expression for the breaking load $q=q_{\max }$ measured in the experiment

$$
q_{\max }=\tau_{a d h} \frac{2 \omega}{h_{a} \lambda_{q}^{2}} \operatorname{coth}\left(\frac{\omega l}{2}\right) .
$$

Formula (17) allows building a graph of dependence of the breaking load $q=q_{\max }$ (measured in the experiment) depending on the various parameters of the model. The captions of the diagrams hereinafter will mean the value of adhesive strength used in the calculation $\tau_{a d h}$. 


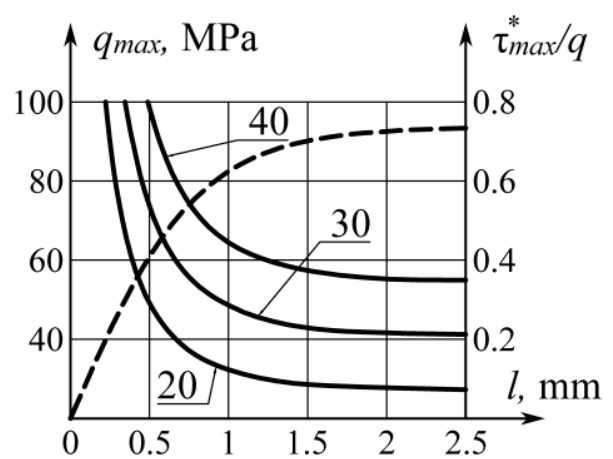

Fig. 6. Theoretical dependences $q_{\max }(l)$ (solid lines) and $\tau_{\max }^{*}(l)$ (dashed line).

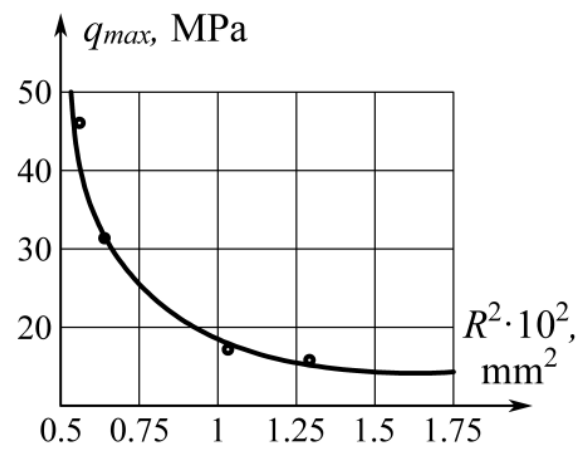

Fig. 7. Experimental dependence $q_{\max }\left(R^{2}\right)$ for cylindrical layered composite.

From Figure 6 it follows that an increase in the length of the bonding area leads to a decrease of breaking load $q_{\max }$ an increase in shear stresses $\left|\tau_{\max }^{*}\right|$. The dependences are asymptotic. Starting with some value (in our case $l=2 \mathrm{~mm}$ ) the length has almost no effect. Similar results (fig. 7) were obtained in the experiment on the test of cylindrical layered composite.

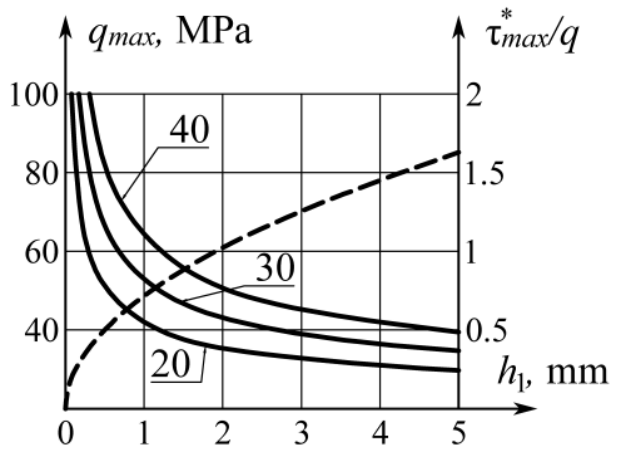

Fig. 8. Theoretical dependences $q_{\max }\left(h_{1}\right)$ (solid lines) and $\tau_{\max }^{*}\left(h_{1}\right)$ (dashed line).

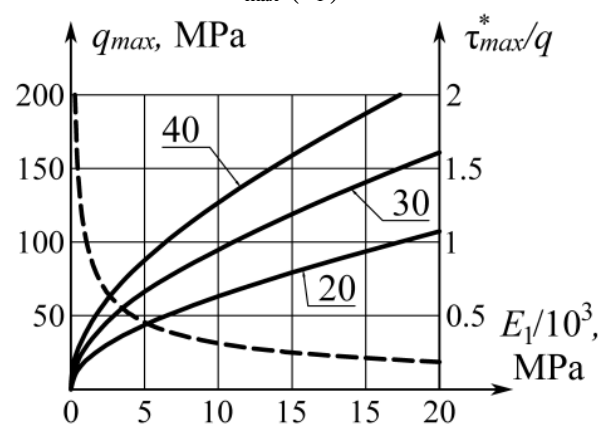

Fig. 10. Theoretical dependences $q_{\max }\left(E_{1}\right)$ (solid lines) and $\tau_{\max }^{*}\left(E_{1}\right)$ (dashed line).

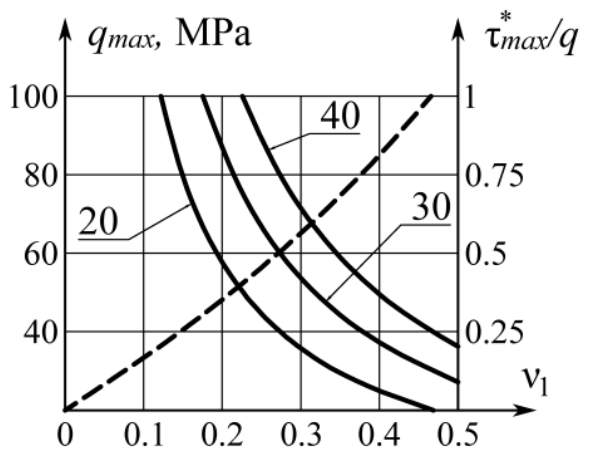

Fig. 9. Theoretical dependences $q_{\max }\left(v_{1}\right)$ (solid lines) and $\tau_{\max }^{*}\left(v_{1}\right)$ (dashed line).

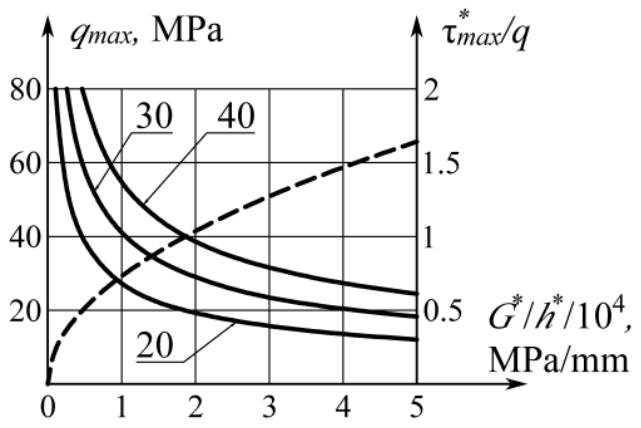

Fig. 11. Theoretical dependences $q_{\max }\left(G^{*} / h^{*}\right)$ (solid lines) and $\tau_{\max }^{*}\left(G^{*} / h^{*}\right)$ (dashed line). 
As we can see, an increase in the thickness (Figure 8) and in Poisson's ratio (Figure 9) of the adhesive layer leads to an increase in shear stresses and, accordingly, a decrease in the magnitude of the breaking load. This is due to the fact that as a result of stretching the adhesive layer in the $z$ direction, due to the Poisson's ratio, its size decreases (compression) in the $x$ and $y$ directions. The greater the Poisson ratio, the more the adhesive is compressed in the $x$ and $y$ directions. The substrate layers prevent compression. The smaller the thickness of the adhesive layer, the easier it is for the substrate layers to prevent compression and the lower are the shear stresses. Reducing the Young modulus of the adhesive layer (Figure 10) increases shear stresses. For the intensity of the adhesive interaction (Figure 11) there is a different picture. The higher the intensity, the higher the shear stresses.

\section{Conclusions}

The calculations show that a non-uniform stress-strain state occurs in the layered composite. Shear stresses reach significant values $\left|\tau_{\max }^{*}\right|$ at the border of the adhesive with a sufficiently large parameter $l$. These shear stresses can exceed the shear adhesive strength $\tau_{\text {adh }}$ of adhesive - substrate pair. This means that in tests for normal separation (transversal strength), in reality, the model can be destroyed by shear stresses. For small values of the parameter $l$, the normal stresses significantly exceed the maximum shear stresses near the edge. In this case, we can find the true transversal strength. Therefore, for the correct experience and evaluation of the transversal strength, it is necessary to pre-evaluate the shear adhesive strength for a given adhesive-substrate pair. This note applies to all model parameters.

The contact layer model applied to the solution of this problem allows us to estimate the influence of each of the parameters on the stress-strain state of the model. To determine the parameters of the contact layer from the experiment [5] it is proposed to use a simplified model. To determine the true adhesive strength of the joint, it is recommended to use a general form model. It gives more accurate results for shear stresses in a narrow zone of the edge effect.

This work was financially supported by the Ministry of Education and Science (state task \#7.6163.2017/6.7).

\section{References}

1. R. Turusov, Mechanics of Composite Materials 50(6), 1119-30 (2014)

2. V. Andreev, R. Turusov, N. Tsybin, MATEC Web of Conferences 117, 00008 (2017)

3. V. Andreev, R. Turusov, N. Tsybin, Procedia Engineering 153, 59-65 (2016)

4. V. Andreev, R. Turusov, N. Tsybin, Procedia Engineering 153, 51-58 (2016)

5. R. Turusov, L. Manevich, Polymer Science, Series D 4(1), 1-4 (2011)

6. R. Turusov, Adhesion mechanics (NRU MGSU, 2015)

7. K. Kaw, Mechanics of composite materials (Taylor \& Francis 2006)

8. V. Vasiliev, E. Morozov, Mech. and analysis of composite materials (Elsevier 2001) 